\title{
Improved electromagnetic bias theory
}

\author{
T. Elfouhaily and D. R. Thompson \\ Applied Physics Laboratory, Johns Hopkins University, Laurel, Maryland
}

\section{B. Chapron}

Département d’Océanographie Spatiale, Institut Français de Recherche pour l'Exploitation de la Mer, Brest France

\author{
D. Vandemark \\ NASA Goddard Space Flight Center, Wallops Island, Virginia
}

\begin{abstract}
In this paper we describe modifications to a previous theory for the altimeter electromagnetic bias (EM bias) of Srokosz [1986]. A major correction introduces a scaling of Srokosz's model by nonnegligible dimensionless ratios that depend on the slope variance of both long and short waves. With these modifications the EM bias is no longer simply a function of the cross skewness between surface elevation and slope but now depends on the variance ratios that represent the modulation between short and long waves. Inclusion of these ratios can reduce previous EM bias estimates by as much as $50 \%$. Different directions for the longwave and shortwave field are also accounted for in the two-dimensional development of our approach. A radar frequency dependence consistent with observation is also predicted by the new model. Derivation extending our development to the next higher order in wave statistics is also presented and discussed.
\end{abstract}

\section{Introduction and Issues}

Altimetry has had an active research role in the past 2 decades, and its usefulness has been recognized in such areas as ocean tides, geodesy, geodynamics, ocean wave height, and surface wind speed. With the advent of TOPEX/Poseidon altimeters the root-mean-square (rms) accuracy of a single-pass sea level measurement is now near $5 \mathrm{~cm}$ [Nouel et al., 1994], thus permitting routine mapping of the circulation dynamics of the ocean surface. The main geophysical parameter that can be inferred by subtraction of the measured satellite altitude from its distance to the Earth's center is the mean sea level (MSL). This measure, although simple in principle, is difficult to realize with high accuracy since satellite ephemeris, atmospheric propagation, and changing surface sea state contaminate altimetric measurements. While orbitographical and atmospheric errors are now near or below the precision requirements because of state-of-the-art positioning systems and radiometry, the electromagnetic bias (EM bias) remains a predominant and uncertain factor in the altimetric error budget. The EM bias exists because for near-nadir electromagnetic scattering, ocean surface waves are stronger reflectors near their troughs than near their crests. This phenomenon leads to an appreciable difference between actual mean sea level and the mean sea level measured by a satellite altimeter. Accurate measurement of actual mean sea level requires an effective and reasonably complete correction for this electromagnetic bias.

Today, the only complete theory of the EM bias is the two-dimensional theory by Srokosz [1986]. To derive this theory, Srokosz applied the weakly nonlincar theory elaborated by Longuet-Higgins [1963] to compute the nonlinear moments of Copyright 2000 by the American Geophysical Union.

Paper number 1999JC900277. 0148-0227/00/1999JC900277\$09.00. the surface using a two-dimensional spectrum. The link between the computed nonlinear moments and the EM bias was guided by an earlier work by Jackson [1979]. This existing EM bias theory relies, however, entirely on the restrictive assumption of the weakly nonlinear theory. In other words, when the conditions of applicability of the weakly nonlinear theory are not satisfied, then Srokosz's theory will not be applicable either.

In a recent study of the EM bias theory [Elfouhaily et al., 1999] we found that this weakly nonlinear theory has been applied outside its domain of applicability in most EM bias simulations [e.g., Jackson, 1979; Glazman et al., 1996]. The problem is caused by the inclusion of short waves (centimeter wavelengths) in the computation of the moment expressions as given by the weakly nonlinear theory. It is well known that wave-wave interactions among short waves are a strong nonlinear process. Barrick and Weber [1977] also demonstrated that a similar breakdown of the weakly nonlinear theory occurs at higher-order statistics when the nonlinear interactions are not weak. In our recent study [Elfouhaily et al., 1999], however, we showed that the inconsistency between imposed and required moments exists even for moments of lower order than predicted by Barrick and Weber [1977] and Barrick and Lipa [1985]. From these mentioned limitations it is clear that Srokosz's theory can be applied only to longer gravity waves in order to avoid the inconsistency in the moments and the violation of weakly nonlinear assumptions. This apparently leaves one with no choice but to exclude short waves, yet these waves are known to have a significant effect on the EM bias [see Rodriguez et al., 1992].

In this paper we propose a theory that includes both short and long waves without causing any incompatibility with weakly nonlinear theories. It will be shown how low- and highorder moments of both short and long waves enter the theory of the EM bias. Among other improvements it will be shown that this new theory is sensitive to the radar frequency as well 
as to the propagation directions of both short and long waves separately.

This paper is organized into three principal parts. The first part provides a succinct review of the literature concerned with the EM bias. There is not sufficient room to cite all papers that have offered useful contributions. Nonetheless, we enumerate the most relevant studies on the subject from both theory and measurement points of view. In the second part we derive a new approach for the EM bias model that ends up being a modification of the previous theory by Srokosz [1986]. This modified theory will be termed the low-order EM bias. In addition, we describe an extension of this theory to include higher-order statistical terms, which we call the high-order EM bias. The third part is concerned with the numerical evaluation of the electromagnetic bias and its comparison with measurements made by the TOPEX altimeter. The last section of the paper is the conclusion followed by three appendices that describe the derivation of the present theory.

\section{Review of the EM bias history}

Since the sixties a weakly nonlinear theory of long gravity waves has existed. This theory aimed to connect wave spectra and statistical moments of the processes that govern the random motion of a water surface. Longuet-Higgins [1963] resolved the hydrodynamic equations to second order by accounting for nonlinear terms. In order to simplify the original complicated problem, Longuet-Higgins chose to make the assumptions of a free surface and undamped waves. By making these assumptions the hydrodynamic equations become analytically resolvable in the sense that no source terms are used for wave generation or wave dissipation. Therefore the coupled differential equations of both surface elevation and velocity potential could be developed to second order in elevation amplitudes. The first order is linear in elevation amplitude and produces Gaussian surface statistics. The second order is quadratic in elevation amplitude and accounts for nonlinear effects that cause the elevation probability density function (pdf) to depart from Gaussianity. The nonlinear behavior is explained by means of wave-wave interactions of couplets among long gravity waves. Through his method, LonguetHiggins expressed the moments of the surface elevation in terms of the spectral density function. The skewnesses of the surface can be interpreted as a double integration of the spectrum for a couplet of wavenumbers nultiplied by a kernel function that predicts the magnitude of the interaction [Elfouhaily et al., 1999]. This weakly nonlinear theory demonstrated that distributions of surface elevations and slopes are in accord with Gram-Charlier developments and with reported measurements by Cox and Munk [1954] and Kinsman [1960].

Theoretical application of Longuet-Higgins [1963] to altimetry was first reported by Jackson [1979] with the goal of finding a theoretical basis for the EM bias earlier measured by Yaplee et al. [1971]. Jackson made a major assumption, in addition to those imposed by the theory of Longuet-Higgins, that surface waves are long crested. This particular assumption modified the problem from two dimensions to only one dimension. Jackson also used the geometrical optics approximation to describe microwave backscatter at nadir. The radar cross section in this context is proportional to the specular point distribution, which in turn depends on the joint pdf of height and slopes claimed to be valid at any scale. Following this logic, Jackson related the altimeter backscatter to the weakly non- linear theory of Longuet-Higgins. In this one-dimensional context the electromagnetic bias $\beta_{E M}$ can be written as

$$
\beta_{\mathrm{LM}}=-\frac{1}{8} \lambda_{12} H_{1 / 3}
$$

where $\lambda_{12}$ is the cross skewness between surtace elevation and slope. $H_{1 / 3}$ is the significant wave height. The numerical evaluation of the skewness parameters was accomplished by using a Phillips-type spectrum (saturated waves) in the LonguetHiggins formulations. Jackson [1979] could, under the liee and undamped wave assumption, estimate the skewness of the surface and therefore the EM bias.

Srokosz [1986] extended the theoretical work of LonguctHiggins [1963] and its application to altimetry by fackson [1979]. In contrast to Jackson [1979], Srokosz [1986] did not assume a long-crested sea. Therefore he had to deal with two-dintensional surface waves. A joint distribution of surface elevation and two slopes was then derived. However, the assumption of free and undamped gravity waves was still required in order to apply the weakly nonlinear theory of Longuet-Higgins. Moreover, the classical assumption of horizontal facets at every scale was applied in order to derive a theoretical expression for the EM bias under a geometrical optics approximation for the EM scattering.

Srokosz [1986] found that the EM bias of a two-dimensional surface can be written in a way similar to Jackson's [1979] expression (1) as

$$
\gamma=\frac{\beta_{E M}}{H_{1 / 3}}=-\frac{1}{8} \frac{\lambda_{120}+\lambda_{102}-2 \lambda_{011} \lambda_{111}}{1-\lambda_{011}^{2}}
$$

Thus the normalized bias is an arithmetic combination involving all cross skewnesses of the two-dimensional surface. Under solely wind-driven conditions, though, $\lambda_{011}$ and $\lambda_{111}$ are zero, in which case the $\gamma$ factor merely becomes [see Lagerloef, 1987]

$$
\gamma=-\frac{1}{8}\left\{\lambda_{120}+\lambda_{102}\right\}
$$

In this case the two-dimensional expression of the EM bias is very similar to the one given by Jackson [1979], with an additional parameter for the cross skewness in the cross-wind direction $\left(\lambda_{102}\right)$. Note also that evaluation of the skewness parameters requires a two-dimensional wavenumber spectrum. A recent paper by Glazman et al. [1996] implements the twodimensional theory of Srokosz [1986]. For their numerical simulation they constructed a two-dimensional spectrum by connbining their unidirectional spectrum [Glazman and Srokosz, 1991] with the spreading function of Apel [1994]. In addition to the wind-wave spectrum, they included a Gaussian spectrum to represent the swell as an extra source of energy. Interaction between swell and wind waves was neglected.

Recent investigations [Elfouhaily et al., 1999] suggest problems in the application of the theory developed by Srokosz [1986]. In particular, that theory is unable to include shortwave effects in the EM bias simulations without generating a serious inconsistency in both low- and high-order moments because of the violation of the conditions under which the weakly nonlinear theory is applicable. This petper presents a modification of Srokosz's theory as well as an extension to higher-order statistics of surface waves. As a result, shortwave statistics can be handled correctly without violation of the weakly nonlinear assumptions. 


\section{Derivation of the Low-Order EMI Bias}

\subsection{Definition of the EM Bias}

The EM bias is defined as the normalized correlation between the radar cross section $\sigma^{0}$ and the surface elevation $\zeta$ :

$$
\beta_{E M}=\frac{\left\langle\zeta \sigma^{0}\right\rangle}{\left\langle\sigma^{0}\right\rangle}=\frac{\iint \zeta \sigma^{0} P\left(\zeta, \sigma^{(0)}\right) d \zeta d \sigma^{0}}{\iint \sigma^{0} P\left(\zeta, \sigma^{(0)}\right) d \zeta d \sigma^{0}}
$$

The correlation in (4) is nonzero only under nonlinear statistics where the slopes and the elevation are correlated. It is clear that if the statistics were Gaussian, then the electromagnetic bias would be zero.

\subsection{Introducing the Long-Wave Tilting}

It is well known [Keller and Wright, 1975; Valenzuela, 1978] that the radar cross section $\sigma^{0}$ is modulated by the tilting slopes, which are correlated with the elevation because of a nonlinear process caused by wave-wave interactions. Now, the ensemble average in (4) can be carried out assuming a known joint pdf of slopes and elevation.

$$
\beta_{E M}=\frac{\iiint \zeta \sigma^{\prime \prime} P_{L}\left(\zeta, \zeta_{x}, \zeta_{y}\right) d \zeta d \zeta_{x} d \zeta_{y}}{\iiint \sigma^{\prime \prime} P_{L}\left(\zeta, \zeta_{x}, \zeta_{y}\right) d \zeta d \zeta_{x} d \zeta_{y}}
$$

The subscript $L$ on the joint pdf of elevation and slopes refers to the longwave tilting. This joint pdf is defined [LonguetHiggins, 1963; Srokosz, 1986] using a Gram-Charlier approximation:

$$
\begin{aligned}
& P_{L}\left(\zeta, \zeta_{x}, \zeta_{y}\right)=\frac{G_{1-\mathrm{D}}(\eta)}{\sqrt{\kappa_{200}}} \frac{G_{2-\mathrm{D}}\left(\eta_{x}^{L}, \eta_{y}^{L} ; \lambda_{011}\right)}{\sqrt{\kappa_{020} \kappa_{002}}} \\
& \cdot\left[1+\frac{\lambda_{300}}{6} H_{300}+\frac{1}{2}\left(\lambda_{120} H_{120}+\lambda_{102} H_{102}+2 \lambda_{111} H_{111}\right)\right],
\end{aligned}
$$

where only orders up to the cross skewness between surface elevation and slopes are retained. The $H_{i j k}$ functions are the generalized Hermite polynomials of the three normalized variables

$$
\eta=\frac{\zeta}{\sqrt{\kappa_{2001}}}, \quad \eta_{x}^{L}=\frac{\zeta_{x}}{\sqrt{\kappa_{020}}}, \quad \eta_{y}^{L}=\frac{\zeta_{y}}{\sqrt{\kappa_{0012}}}
$$

and the cross correlation $\lambda_{011}$ between the $x$ and $y$ components of the slopes. In (6) the one-and two-dimensional Gaussians are symbolized by $G_{1-D}$ and $G_{2-D}$ (see Appendix A), respectively. The three-dimensional Hermite polynonials can be reduced to the product of a one-dimensional and a twodimensional Hermite:

$$
H_{i j k}\left(\eta, \eta_{x}^{L}, \eta_{y}^{L}, \lambda_{011}\right)=H_{i}(\eta) H_{j k}\left(\eta_{x}^{L}, \eta_{y}^{L}, \lambda_{011}\right) .
$$

Definition and expressions of the generalized two-dimensional Hermite polynomials are detailed in Appendix A.

\subsection{Introducing Scattering by Short Waves}

In a geometric optics (GO) assumption the radar cross section is proportional to the slope pdf of short waves expressed in terms of local tilting over long waves:

$$
\beta_{L M}=\frac{\iiint \zeta_{s}\left(\zeta_{x}, \zeta_{y}\right) P_{L}\left(\zeta, \zeta_{x}, \zeta_{y}\right) d \zeta_{\zeta} d \zeta_{x} d \zeta_{y}}{\iiint P_{s}\left(\zeta_{x}, \zeta_{y}\right) P_{L}\left(\zeta, \zeta_{x}, \zeta_{y}\right) d \zeta_{\zeta} d \zeta_{x} d \zeta_{y}}
$$

According to (9), (6), and (8) the integration over elevation can be carried out without explicit knowleclge of $P_{S}$ since this function depends only on the slope components. At this point the major difference between our formulation and Srokosz's theory is that the critical assumption of horizontal facets at every scale is not made in our theory even though the geometric optics assumption is retained.

After integration over the elevation the original triple integrals (equation (9)) now reduce to double integrals over the slope components only. Hence the electromagnetic bias becomes

$\beta_{E M}=\frac{\iint P_{S}\left(\zeta_{x}, \zeta_{y}\right) G_{2-D}^{L} Q_{2-D}^{L}\left(\eta_{x}^{L}, \eta_{y}^{L}\right) d \zeta_{. x} d \zeta_{y}}{\iint P_{S}\left(\zeta_{x}, \zeta_{y}\right) G_{2-D}^{L} d \zeta_{x} d \zeta_{y}} \sqrt{\kappa_{2(n+1 .}}$

The longwave tilting factors $G_{2-\mathrm{D}}^{L}$ and $Q_{2-\mathrm{D}} L$ are the twodimensional Gaussian pdf and the longwave coupling due to nonlinear statistics:

$$
G_{2-\mathrm{D}}^{L}=\frac{1}{\sqrt{\kappa_{020} \kappa_{002}}} G_{2-\mathrm{D}}\left(\eta_{x}^{L}, \eta_{y}^{L} ; \lambda_{011}\right)
$$

$Q_{2-\mathrm{D}}^{L}=\frac{1}{2} \lambda_{120} H_{20}\left(\eta_{r}^{L}, \eta_{y}^{L} ; \lambda_{0111}\right)+\frac{1}{2} \lambda_{102} H_{02}+\lambda_{111} H_{11}$.

Arguments of the generalized Hermite polynomials in the right-hand side of this equation were dropped for brevity.

To simplify, let the slope pdf of short waves be consiclered as a simple Gaussian distribution since the nonlinear order retained is only the cross skewness between the elevation and the slopes. The generalized expression for $P_{S}$ is then

$$
P_{s}\left(\zeta_{x}, \zeta_{y}\right)=\frac{1}{\sqrt{\kappa_{20} \kappa_{02}}} G_{2-\mathrm{D}}\left(\eta_{x}^{s}, \eta_{y}^{s} ; \lambda_{11}\right),
$$

where the slope components are normalized by the rms slope of short waves:

$$
\begin{gathered}
\eta_{x}^{S}=\frac{\zeta_{x}}{\sqrt{\kappa_{20}}} \\
\eta_{y}^{S}=\frac{\zeta_{y}}{\sqrt{\kappa_{02}}} .
\end{gathered}
$$

By replacing $P_{S}$ by expression (12) in (10), we can rewrite (13b) as

$$
\beta_{E M}=\frac{\iint G_{2-\mathrm{D}}^{S} G_{2-\mathrm{D}}^{L} Q_{2-\mathrm{D}}^{L}\left(\eta_{x}^{L}, \eta_{y}^{L}\right) d \zeta_{x} d \zeta_{y}}{\iint G_{2-\mathrm{D}}^{S} G_{2-\mathrm{D}}^{L} d \zeta_{x} d \zeta_{y}} \sqrt{\kappa_{200}}
$$

The product of longwave and shortwave Gaussians can now be merged into one single two-dimensional Gaussian to give 


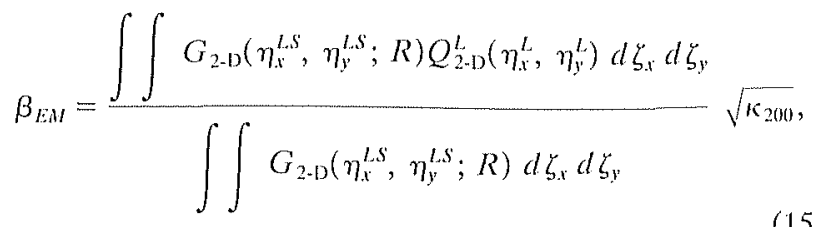

where the normalized coupled longwave and shortwave slopes are

$$
\eta_{.:}^{L S}=\frac{\zeta_{x}}{\sigma_{x}} \eta_{y}^{L S}=\frac{\zeta_{y}}{\sigma_{y}} .
$$

The resulting variables from this merger of two Gaussians are given by a nonlinear system of equations:

$$
\begin{aligned}
\frac{1}{\sigma_{x}^{2}\left(1-R^{2}\right)}= & \frac{1}{\kappa_{020}\left(1-\lambda_{011}^{2}\right)}+\frac{1}{\kappa_{20}\left(1-\lambda_{11}^{2}\right)} \triangleq \alpha_{x}^{2}, \\
\frac{1}{\sigma_{y}^{2}\left(1-R^{2}\right)}= & \frac{1}{\kappa_{0102}\left(1-\lambda_{011}^{2}\right)}+\frac{1}{\kappa_{012}\left(1-\lambda_{11}^{2}\right)} \stackrel{\Delta}{\triangleq} \alpha_{y}^{2}, \\
\frac{R}{\sigma_{x} \sigma_{y}\left(1-R^{2}\right)}= & \frac{\lambda_{011}}{\sqrt{\kappa_{0120} \kappa_{0102}}\left(1-\lambda_{011}^{2}\right)} \\
& +\frac{\lambda_{11}}{\sqrt{\kappa_{20} \kappa_{02}}\left(1-\lambda_{11}^{2}\right)} \stackrel{\Delta}{=} r .
\end{aligned}
$$

Inversion of this nonlinear system is complicated. For this reason our analytical solution is given only in terms of the dummy variables that are at the right-hand side of the previous equations, namely, $\alpha_{x}, \alpha_{y}$, and $r$. This inversion gives

$$
\begin{gathered}
\sigma_{x}=\frac{\alpha_{y}}{\sqrt{\alpha_{x}^{2} \alpha_{y}^{2}-r^{2}}}, \\
\sigma_{y}=\frac{\alpha_{x}}{\sqrt{\alpha_{x}^{2} \alpha_{y}^{2}-r^{2}}}, \\
R=\frac{r}{\alpha_{x} \alpha_{y}} .
\end{gathered}
$$

The new variable $R$ can be understood as a generalized coupling coefficient that carries information from both crosscorrelation coefficients, $\lambda_{011}$ of long waves and $\lambda_{11}$ of short waves. The actual expression for $R$ is a complicated function of $\lambda_{011}$ and $\lambda_{11}$ together with the $x$ and $y$ components of slope variances of both long and short waves. It should be noted at this point that $R$ is symmetric in its $x$ and $y$ components. This property will be used intensively in the following sections.

\subsection{Computation of the Electromagnetic Bias}

Evaluating the double integral in (15) could be an overwhelming task if one tries to solve the double integrals without further consideration of the generalized Gaussian distribution. In fact, the two-dimensional Gaussian distribution is formed from two coupled one-dimensional Gaussians. A simple change of variable helps to decouple the integrals into their Gaussians and leaves the coupling to the $Q_{2 \text {-D }}^{L}$ function alone. After changing the variables of integration in (15) one can simply write the electromagnetic bias expression as one double integral:

$$
\begin{aligned}
\beta_{E M}= & \iint G_{2-\mathrm{D}}(X, Y ; 0) Q_{2-\mathrm{D}}^{L} \\
& \cdot\left[\Delta_{x}^{L}\left(X \sqrt{1-R^{2}}+R Y\right), \Delta_{y}^{L} Y\right] d X d Y,
\end{aligned}
$$

where the change of variables is apparent in the arguments of $Q_{2-\text { di }}^{L}$. The Jacobian of the transtormation cancels with the denominator in (15). To simplify the notation, we introduce two dimensionless quantities that will be used throughout this work,

$$
\Delta_{x}^{L}=\frac{\sigma_{x}}{\sqrt{\kappa_{021}}} \Delta_{y}^{L}=\frac{\sigma_{y}}{\sqrt{\kappa_{0102}}} .
$$

The superscript $L$ in (20) indicates that the normalization is referenced to the rms of longwave tilting. The reader should be aware that the superseript $L$ does not mean that $\Delta_{x}^{L}$ and $\Delta_{y}^{L}$ are functions only of longwave parameters. Indeed, the parameters $\sigma_{x}$ and $\sigma_{y}$ involve both longwave and shortwave moments. After substitution by the expression of $Q_{2-\mathrm{D}}^{L}$ (equation (11b)) into (19), one can cast the final form of the electromagnetic bias as

$$
\beta_{E M}=\frac{\sqrt{\kappa_{200}}}{2}\left(\lambda_{120} W_{20}+\lambda_{102} W_{02}+2 \lambda_{111} W_{11}\right)
$$

where the weighting functions, which multiply the cross skewnesses, are defined as

$$
\begin{aligned}
W_{m n}= & \iint G_{1-\mathrm{D}}(X) G_{1-\mathrm{D}}(Y) H_{m n} \\
& \cdot\left[\Delta_{Y}^{L}\left(X \sqrt{1-R^{2}}+R Y\right), \Delta_{y}^{L} Y\right] d X d Y .
\end{aligned}
$$

Analytical expressions of the weighting functions can be computed by expanding the generalized Hermite polynomials in terms of powers of $X$ and $Y$ and by using the following moment properties of a one-dimensional Gaussian:

$$
\begin{gathered}
\int_{-\infty}^{+\infty} x^{2 n+1} G_{1-\mathrm{D}}(x) d x=0 \\
\int_{-\infty}^{+\infty} x^{2 n} G_{1-\mathrm{D}}(x) d x=(2 n-1) ! !
\end{gathered}
$$

The double factorial in (23) is a mathematical notation to indicate that the factorial product is performed on either even or odd numbers. In this case the double factorial is always performed on odd number up to $(2 n-1)$. Powers of $X$ and $Y$ in (22) can simply be replaced by their values in (23). This makes the evaluation of the originally complicated double integral very simple. Unfortunately, the same comment cannot be made for the final expression of these weighting functions.

\subsection{Final Analytical Expression of the Low-Order EM Bias}

It is convenient to write the EM bias as a quantity normalized by the significant wave height $\left(H_{1 / 3}=4 \sqrt{\kappa_{200}}\right)$ :

$$
\gamma=\frac{\beta_{E M}}{H_{1 / 3}}=\frac{1}{8}\left(\lambda_{120} W_{20}+\lambda_{102} W_{02}+2 \lambda_{111} W_{11}\right)
$$

Equation (24) forms the final analytical expression of the normalized EM bias that includes nonlinearities up to the cross skewness between the elevation and the slopes. After some tedious algebraic manipulations the weighting functions can be written in a compact form:

$$
W_{20}=H_{20}\left(\Delta_{x}^{L}, \Delta_{y}^{L} ; \lambda_{011}\right)+\frac{2 \lambda_{011}}{1-\lambda_{011}^{2}} \frac{1-R}{1-\lambda_{011}^{2}} \Delta_{x}^{L} \Delta_{y}^{L}
$$




$$
W_{11}=H_{11}\left(\Delta_{x}^{L}, \Delta_{y}^{L} ; \lambda_{011}\right)-\frac{1+\lambda_{011}^{2}}{1-\lambda_{011}^{2}} \frac{1-R}{1-\lambda_{011}^{2}} \Delta_{x}^{L} \Delta_{y}^{L},
$$

where the expression for $W_{02}$ can be obtained from that of $W_{20}$ by simply interchanging the $x$ and $y$ subscripts. It should be noted that the cross-correlation moments $\lambda_{011}$ and $\lambda_{11}$ and the generalized coupling coefficient $R$ are symmetric and therefore are invariant to the interchange of $x$ and $y$ components.

It is obvious that this new theory is sensitive to directions of both short and long waves. In fact, $\lambda_{01}$, carries directionality information of long waves in a manner explained and already exploited by Srokosz [1986, 1987] and Glazman et al. [1996]. An additional directionality parameter, $\lambda_{11}$ for the short waves, is now present in this formulation. So, shortwave and longwave directions are now accounted for separately. For instance, short wind waves are most often aligned to the direction of the wind. Very rarely are long waves aligned to the wind, which makes theories with a single directional parameter less realistic. Another nice feature of this theory is the sensitivity to the radar frequency coming through the frequency cutoff imposed on the rms slopes of short waves under EM scattering theory. Indeed, Brown [1978] showed that slope variances involved in geometric optics models are filtered in frequency to some multiple of the electromagnetic frequency transmitted by the radar. Usually the cutoff wavelength is chosen to be about three times higher than the electromagnetic wavelength [see also Thompson, 1988; Chapron et al., 1995].

Interaction between long and short waves is also represented in this model by the delta parameters in (25). It can be shown that these deltas are in fact approximate measures of the hydrodynamic interactions between short and long waves, which can also be seen as surrogates of higher-order statistics. Elfouhaily [1996, paragraph III.6] clearly showed that a connection can be made between the ratios (presently named deltas) and a hydrodynamic-like modulation of short waves by the orbital velocities of longer ones.

\section{Special Illustrative Cases}

The present theory encompasses previous EM bias theories as special cases. One can show that when short waves are neglected, Srokosz's [1986] theory can be retrieved. When directionality information is ignored, our formulation converges toward Jackson's [1979]. Another interesting limit is the parallel to Srokosz's theory where the cross-correlation of long waves is neglected.

\subsection{Srokosz's Theory Solely Relying on Long Waves}

Srokosz's [1986] theory is actually a special case in our derivation when the shortwave effect is eliminated by letting both $\Delta_{x}^{L}$ and $\Delta_{y}^{L}$ tend to zero. The weighting functions in this special case become (see Appendix A)

$$
\begin{gathered}
\lim _{\Delta_{x}^{t}, \Delta_{y}^{\prime} \rightarrow 01} W_{20}=H_{20}\left(0,0 ; \lambda_{011}\right)=-\frac{1}{1-\lambda_{011}^{2}} \\
\lim _{\Delta_{x}^{l}, \Delta_{y}^{L} \rightarrow 0} W_{11}=H_{11}\left(0,0 ; \lambda_{011}\right)=\frac{\lambda_{011}}{1-\lambda_{011}^{2}} .
\end{gathered}
$$

These weights are now pure functions of the cross correlation $\lambda_{011}$ with no dependence on the slope variances of both short and long waves. In other words, the expression of the normal- ized bias, for this limit and as presented by Srokosz, is independent of the slope variance of either short or long waves. If one uses these limits in computing (24), one gets

$$
\gamma^{(1) 1)}=\lim _{\left.a_{1}^{t}, A_{s}^{t} \rightarrow 0\right)} \gamma=-\frac{1 \lambda_{120}+\lambda_{102}-2 \lambda_{111} \lambda_{011}}{8},
$$

which is identical to the expression given by Srokosz [1986] and recalled in (2).

\subsection{Parallel to Srokosz's Theory From the Shortwave Standpoint}

A parallel to Srokosz's theory can be found when only the directionality of short waves is considered. Let the cross correlation $\lambda_{01}$, tend to zero while keeping the cross correlation of short waves $\lambda_{11}$ in its general form. The weighting functions for this limit become

$$
\begin{aligned}
& \lim _{\lambda_{i+1} \rightarrow 0} W_{20}=H_{20}\left(\Delta_{x}^{L}, \Delta_{y}^{L} ; 0\right)=H_{2}\left(\Delta_{x}^{L}\right) \\
& \lim _{\substack{(m, 1 \rightarrow 0\\
}} W_{11}=H_{11}\left(\Delta_{x}^{L}, \Delta_{y}^{L} ; 0\right)-(1-R) \Delta_{x}^{L}, \Delta_{y}^{L}=R \Delta_{x}^{L} \Delta_{y^{\prime}}^{L}
\end{aligned}
$$

When these limits are introduced in (24), we obtain

$$
\gamma_{0}=\lim _{\left.\lambda_{1011} \rightarrow 0\right)} \gamma=\frac{1}{8}\left[\lambda_{120} H_{2}\left(\Delta_{x}^{L}\right)+\lambda_{112} H_{2}\left(\Delta_{y}^{L}\right)+2 \lambda_{111} R \Delta_{x}^{L} \Delta_{y}^{L}\right] .
$$

This special case of the EM bias can be very handy and tractable for implementation without losing the generality of the problem [Lagerloef', 1987]. Indeed, it can always be assumed that the principal axis of the observer is aligned with the long waves. In this case, $\lambda_{011}$ goes to zero while $\lambda_{11}$ stays as an arbitrary parameter reflecting the change in the direction of short waves [see Lagerloef, 1987]. This simplified model also keeps the sensitivity to the radar frequency in addition to the longwave-shortwave interaction represented by the delta parameters.

\subsection{Simplest But Most Insightful Special Case}

Now let both $\lambda_{011}$ and $\lambda_{11}$ tend to zero and compare the result with the equivalent simplification proposed by Lagerloef [1987] to the Srokosz formulation. When these limits are applied, the deltas become

$$
\begin{aligned}
& \delta_{x}^{L}=\lim _{\lambda_{011,} \lambda_{11} \rightarrow 0} \Delta_{x}^{L}=\sqrt{\frac{\kappa_{20}}{\kappa_{020}+\kappa_{20}}} \\
& \delta_{y}^{L}=\lim _{\lambda_{111, \lambda_{11} \rightarrow 0}} \Delta_{y}^{L}=\sqrt{\frac{\kappa_{012}}{\kappa_{0102}+\kappa_{02}}},
\end{aligned}
$$

which are very simple arithmetic functions of longwave and shortwave variances of the slopes. The simplest EM bias expression generated by this expression after substitution of (30) into (24) turns out to be

$$
\begin{aligned}
& \gamma_{(00}=\lim _{\left.\lambda_{011}, \lambda_{11} \rightarrow 0\right)} \gamma=\frac{1}{8}\left[\lambda_{120} H_{2}\left(\delta_{x}^{L}\right)+\lambda_{102} H_{2}\left(\delta_{y}^{L}\right)\right] \\
& \gamma_{000}=\lim _{\lambda_{\left(11, \lambda_{11} \rightarrow 0\right)}} \gamma=-\frac{1}{8}\left\{\lambda_{120} \frac{\kappa_{020}}{\kappa_{(120)}+\kappa_{20}}+\lambda_{102} \frac{\kappa_{(012}}{\kappa_{(0102}+\kappa_{(12}}\right\} .
\end{aligned}
$$

This simplified theory is to be compared with (3), the simplified case of Srokosz's theory, which is reproduced hereafter by 


$$
\gamma_{(0)}^{(101)}=\lim _{\lambda(101, \rightarrow 0)} \gamma^{(010}=-\frac{1}{8}\left(\lambda_{120}+\lambda_{102}\right)
$$

The difference between (31) and (32) is clear. The crossskewness parameters in our formulation are weighted by the ratio between the slope variance of long waves and the total slope variance. These ratios are typically of the order of 0.5 and therefore cannot be neglected. Moreover, the presence of the shortwave slope variance in the ratios will induce sensitivity of this EM bias expression to the radar frequency [Brown, 1978; Chapron et al., 1995]. This result points out to the reader that the comments made in the literature about the need for an EM model other than the geometric optics in order to capture the frequency sensitivity must be revised [e.g., Amold et al., 1990, 1991; Rodriguez et al., 1992]. The consensus from our theory is that short waves are responsible for the frequency dependence. More importantly, it appears from our formulation that the modulation of short waves by long waves is the principal driver, through the weighting functions, of the EM bias.

\section{Derivation of the High-Order EM Bias}

We showed above that the earlier theories, such as the one developed by Srokosz [1986], were lacking information from lower-order statistics represented by ratios of the slope variances of both long and short waves. The addition of the shortwave effects can be interpreted as a modification to Srokosz's theory. A remaining question is whether higher-order statistics, higher than the cross skewness between elevation and slopes, could have an effect on the electromagnetic bias? In this section we present an extension of the present EM bias formulation to include higher-order statistics.

First, we begin by extending the joint pdf of long waves (equation (6)) to higher Hermite polynomials. The extension will be limited to the next higher nonlinear order, which is the cross kurtosis between the elevation and the slopes,

$$
\begin{aligned}
P_{L}\left(\zeta, \zeta_{x}, \zeta_{y}\right)=\frac{G_{1-\mathrm{D}}(\eta)}{\sqrt{\kappa_{200}}} \frac{G_{2-\mathrm{D}}\left(\eta_{x}^{L}, \eta_{y}^{L} ; \lambda_{011}\right)}{\sqrt{\kappa_{020} \kappa_{0102}}} \\
\cdot\left[1+\frac{\lambda_{300}}{6} H_{300}+\frac{1}{2}\left(\lambda_{120} H_{120}+\lambda_{102} H_{1112}+2 \lambda_{111} H_{111}\right)\right. \\
\left.\cdot \frac{1}{2}\left(\lambda_{130} H_{130}+\lambda_{103} H_{103}\right)+\frac{1}{2}\left(\lambda_{121} H_{121}+\lambda_{112} H_{112}\right)\right]
\end{aligned}
$$

The definition and expression of the higher-order Hermite polynomials are given in Appendix A. We also derive some necessary identities in Appendix B.

In parallel the shortwave pdf (equation (12)) of the slope is extended to include the next nonlinear order, which is the skewness of the slopes,

$$
\begin{aligned}
& P_{S}\left(\zeta_{x}, \zeta_{y}\right)=\frac{G_{2-\mathrm{D}}\left(\eta_{x}^{S}, \eta_{y}^{S} ; \lambda_{11}\right)}{\sqrt{\kappa_{20} \kappa_{02}}} \\
& \cdot\left[1+\frac{1}{6}\left(\lambda_{30} H_{30}+\lambda_{03} H_{03}\right)+\frac{1}{2}\left(\lambda_{21} H_{21}+\lambda_{12} H_{12}\right)\right] .
\end{aligned}
$$

Now we introduce these extensions of the joint pdfs of both long (equation (33)) and short (equation (34)) waves in to the definition of the electromagnetic bias in (9). We obtain, after integration over the elevation, the equivalent of $(10)$ as

$$
\beta_{L M}=\frac{\iint G_{2-\mathrm{D}}\left(\eta_{x}^{L S}, \eta_{y}^{L S} ; R\right) Q_{2-\mathrm{D}}^{L}\left(\eta_{x}^{L}, \eta_{y}^{L}\right) Q_{2-\mathrm{D}}^{S}\left(\eta_{x}^{S}, \eta_{y}^{S}\right) d \zeta_{x} d \zeta_{y}}{\iint G_{2-\mathrm{D}}\left(\eta_{x}^{L S}, \eta_{y}^{L S} ; R\right) d \zeta_{x} d \zeta_{y}}
$$$$
\cdot \sqrt{\kappa_{200}}
$$

where the longwave and shortwave couplings due to higher nonlinear orders are represented by $Q_{2-D}^{L}$ and $Q_{2-D}^{S}$, respectively. Their expressions are

$$
\begin{aligned}
& Q_{2-\mathrm{D}}^{L}\left(\eta_{x}^{L}, \eta_{y}^{L}\right)=\frac{1}{2}\left(\lambda_{120} H_{20}+\lambda_{102} H_{02}\right)+\lambda_{111} H_{11} \\
& \quad+\frac{1}{6}\left(\lambda_{130} H_{130}+\lambda_{1013} H_{103}\right)+\frac{1}{2}\left(\lambda_{121} H_{121}+\lambda_{112} H_{112}\right) \\
& Q_{2-\mathrm{D}}^{S}\left(\eta_{x}^{S}, \eta_{y}^{S}\right)=\frac{1}{6}\left(\lambda_{301} H_{30}+\lambda_{03} H_{03}\right)+\frac{1}{2}\left(\lambda_{21} H_{21}+\lambda_{12} H_{12}\right)
\end{aligned}
$$

After normalization of the electromagnetic bias by the significant wave height the total EM bias that includes both low and high orders can be rewritten into the compact form

$$
\begin{aligned}
\gamma= & \frac{1}{8}\left(\lambda_{121} W_{20}+\lambda_{102} W_{02}+2 \lambda_{111} W_{11}+\lambda_{30} \lambda_{130} W_{30}^{30}\right. \\
& \left.+\lambda_{013} \lambda_{103} W_{03}^{03}+\lambda_{21} \lambda_{121} W_{21}^{21}+\lambda_{12} \lambda_{112} W_{12}^{12}\right)
\end{aligned}
$$

where $W_{i j}$ are the low-order weighting functions derived above (equation (25)). The $W_{i j}^{m n}$ factors in (37) are the higher-order weighting functions that multiply the slope skewness of short waves and the cross kurtosis between short and long waves. These higher-order weighting functions are defined as

$$
\begin{aligned}
& W_{i j}^{h l}=\frac{1}{i ! j !} \\
& \cdot \frac{\iint G_{2-\mathrm{D}}\left(\eta_{x}^{L S}, \eta_{y}^{L S} ; R\right) H_{i j}\left(\eta_{x}^{L}, \eta_{y}^{L} ; \lambda_{011}\right) H_{k l}\left(\eta_{x}^{S}, \eta_{y}^{S} ; \lambda_{\mathrm{II}}\right) d \zeta_{x} d \zeta_{y}}{\iint G_{2-\mathrm{D}}\left(\eta_{x}^{L S}, \eta_{y}^{L S} ; R\right) d \zeta_{x} d \zeta_{y}},
\end{aligned}
$$

where a change of variable is recommended in order to decouple the two-dimensional Gaussian to permit analytical evaluation of these functions (see Appendix C). Needless to say, these weighting functions are very complicated expressions under general conditions. However, in the particular case where both cross-correlation coefficients $\lambda_{001}$ and $\lambda_{11}$ are zero the higher-order weighting functions become simply

$$
W_{30}^{30}=\frac{1}{6}\left(5 H_{30}^{L} H_{30}^{S}+12 H_{30}^{L} H_{10}^{S}+12 H_{30}^{S} H_{10}^{L}+30 H_{10}^{L} H_{10}^{S}\right)
$$

$W_{21}^{21}=\frac{1}{2}\left\{3 H_{21}^{L} H_{21}^{S}+2 H_{21}^{L} H_{01}^{S}+2 H_{21}^{S} H_{01}^{L}+2 H_{01}^{L} H_{01}^{S}\right\}$.

Appendix C shows that the coefficients of the double Hermite polynomials are constants only under the simplifying condition of zero cross-correlations.

Taking advantage of the compact form of the delta parameters in (30), we can simplify further the higher-order weighting functions and write 


$$
\begin{gathered}
W_{30}^{30}=\frac{5}{6}\left(\delta_{x}^{L}\right)^{3}\left(\delta_{x}^{S}\right)^{3}=\frac{5}{6}\left(\frac{\sqrt{\kappa_{020} \kappa_{201}}}{\kappa_{020}+\kappa_{20}}\right)^{3} \\
W_{21}^{21}=\frac{3}{2} 3\left(\delta_{x}^{L}\right)^{2}\left(\delta_{y}^{S}\right)^{2} \delta_{y}^{L} \delta_{y}^{S}=\frac{3}{2} \frac{\kappa_{0202} \kappa_{20}}{\left(\kappa_{0120}+\kappa_{20}\right)^{2}} \frac{\sqrt{\kappa_{002} \kappa_{02}}}{\kappa_{0102}+\kappa_{02}} .
\end{gathered}
$$

Similar to the low-order derivation, the high-order weights are also ratios of the slope valiances of long and short waves. It is interesting to note that the low moments, such as the slope variances, also enter in the expression of the high-order EM bias alongside the slope skewness of short waves and the cross kurtosis.

The simplified expression of the normalized EM bias in (31) is, finally, extendable to

$$
\begin{aligned}
\gamma_{00}= & \frac{1}{8}\left[-\lambda_{120} \frac{\kappa_{(120)}}{\kappa_{020}+\kappa_{21}}+\lambda_{130} \lambda_{30} \frac{5}{6}\left(\frac{\sqrt{\kappa_{020} \kappa_{20}}}{\kappa_{020}+\kappa_{20}}\right)^{3}\right. \\
& \left.+\lambda_{121} \lambda_{21} \frac{3}{2} \frac{\kappa_{(120} \kappa_{21}}{\left(\kappa_{020}+\kappa_{20}\right)^{2}} \frac{\sqrt{\kappa_{(102} \kappa_{02}}}{\kappa_{0102}+\kappa_{02}}+(x \rightleftharpoons y)\right] .
\end{aligned}
$$

This compact form can be obtained only when cross correlations are set to zero; otherwise, the final expression of the total normalized EM bias is much more complicated and will not be explicitly given in this paper (see Appendix C for guidance).

\section{Liscussion}

The low-order bias depends on both the weighting functions and the cross-skewness moments. A numerical simulation of the former is quite easy because it relies essentially on the variances of long and short waves. The cross-skewness factors are more difficult to compute and require the use of expressions from the weakly nonlinear theory as developed by Srokosz [1986] and simulated by Glazman et al. [1996]. The cross skewness is generated by three-wave interactions among gravity waves. For this reason only the weakly nonlinear theory of Longuet-Higgins [1963] is applicable though with some limitations [see Elfouhaily et al., 1999]. The high-order EM bias is somewhat similar to the lower-order one in the sense that it depends on both higher-order statistics and some weighting functions. The major difference is that the higher-order EM bias depends on statistics of higher order than the cross skewness, namely, the slope skewnesses of short waves and the cross kurtosis between elevation and slopes. Unfortunately, no existing theory describes these higher-order statistics. A numerical evaluation of the high-order EM bias is thus premature. The skewness of the slopes and the cross kurtosis are generated by four-wave interactions among gravity waves, which cannot be represented by the weakly nonlinear theory of Longuet-Higgins [1963]. Several other theories do handie fourwave interactions, or even higher interactions, in the gravity wave domain. These existing theories do not, however, provide the bridge between the wave coupling and the nonlinear statistics. Recently [Elfouhaily et al., 2000], a demonstration was provided on how the Longuet-Higgins's [1963] theory may be extended to more general theories such as the surface perturbation approach by Hasselmamn [1961] and the Hamiltonian theory by Zakharov [1968]. This kind of generalization is needed in order to evaluate the skewness of the slopes and the cross kurtosis, which in turn permits the evaluation of the higher-order EM bias. It is not the scope of the current paper to extend the nonlinear theory of Longuet-Higgins [1963] for

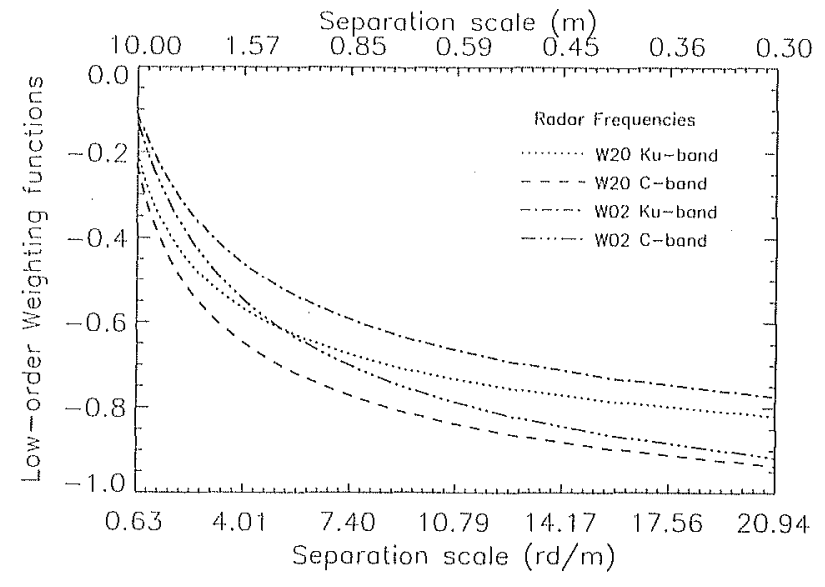

Figure 1. Numerical evaluation of the low-order weighting functions ( $W_{20}$ and $W_{02}$ ) at two different radar frequencies, $\mathrm{Ku}$ band $(2 \mathrm{~cm})$ and $\mathrm{C}$ band $(6 \mathrm{~cm})$, when both short and long waves are aligned to the principal axis $\left(\lambda_{011}\right.$ and $\left.\lambda_{11}=0\right)$ using the two-dimensional spectrum by Elfoulhaily et al. [1997]. One wind speed is considered $\left(U_{10}=7 \mathrm{~m} \mathrm{~s}^{-1}\right)$ for an almost fully developed sea (inverse wave age $\Omega=1$ ). The bottom $x$ axis depicts the separation wavenumber in $\mathrm{id} \mathrm{m}^{-1}$, while the top axis gives the separation wavelength in meters. The highest frequency considered is called the cutoff frequency, which is $\sim 3$ times less than the considered electromagnetic (EM) frequency. Note that the magnitude of the Ku band response is always larger than that of the $\mathrm{C}$ band.

inclusion of four-wave interactions. Instead, we will only evaluate weighting functions as well as the EM bias for the low order as expressed by our new formulation and compare the new formulation with the previous formulation by Srokosz [1986] and with the measured EM bias. Our evaluations suggest that the low-order solution provides a substantial modification of past EM bias simulations. It is also clear that higherorder terms will act as corrections without complete alteration of low-order results.

\section{Numerical Evaluation of the Low-Order EM bias}

\subsection{Weighting Functions}

We first start by a numerical evaluation of the low-order weighting functions ( $W_{20}$ and $W_{02}$ ) under the special case $\lambda_{011}$ and $\lambda_{11}=0$ for the sake of simplicity. The simulation results are shown in Figure 1. Two different radar frequencies Ku band $(2 \mathrm{~cm})$ and $\mathrm{C}$ band $(6 \mathrm{~cm})$ are considered, which in turn determine the high-frequency cutoff for which the shortwave slope variances are computed. It is generally believed that the cutoff should be 3 times the EM wavelength [Brown, 1978; Chapron et al., 1995]. Our directional spectrum [Elfouhaily et al., 1997] is used to realize the numerical evaluation for both $x$ and $y$ components of the slope variance. Only one wind speed is considered $\left(U_{10}=7 \mathrm{~m} \mathrm{~s}^{-1}\right)$ for an almost fully developed sea (inverse wave age $\Omega=1$ ). The curves are presented as a function of the separation scale. When the separation scale is in the centimeter range, the weighting functions approach -1 , which converges to the same limit of Srokosz's theory. The curve representing the $\mathrm{Ku}$ band weighting function is always higher (lower in absolute value because of the minus sign) than that of the $\mathrm{C}$ band in Figure 1. For both radar frequencies, 


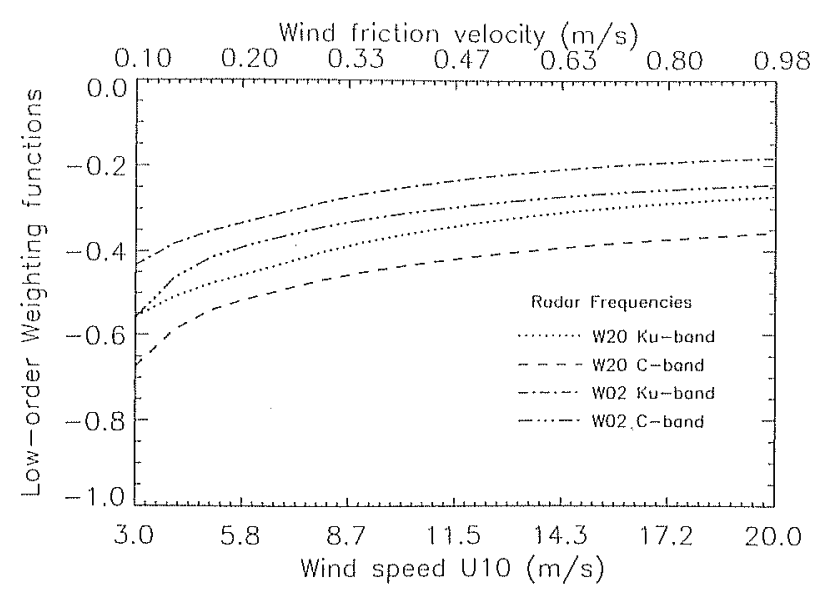

Figure 2. Same as Figure 1 but now with a fixed separation taken to be 10 times the dominant peak wavenumber $\left(k_{s}=10\right.$ $\left.k_{p}\right)$. The wind speed is varied from 3 up to $20 \mathrm{~m} \mathrm{~s}^{-1}$ for an inverse wave age $\Omega=1$. Note that the magnitude of the Ku band weighting functions are always higher than those for the $\mathrm{C}$ band. The absolute value of the weighting functions gets smaller with increasing wind speeds.

however, the low-order weighting functions always reduce the total EM bias by almost a factor of $\frac{1}{2}$ when the separation scale is in the intermediate wave region (wavelength between 1 and $10 \mathrm{~m}$ ).

A sensitivity study is also conducted on the weighting functions to quantify their clependence on the wind speed and wave development. Figure 2 depicts the weighting functions assuming a wind-dependent separation scale. The separation scale is taken to be proportional to the dominant peak frequency. Donelan and Pierson [1987] suggested that 10 times the peak wavenumber is a natural separation scale between long and short waves [see also Elfouhaily et al., 1997]. Figure 2 also shows that the absolute value of the weighting functions decreases with increasing wind speeds. The same monotonic behavior of the weighting functions can be seen in Figure 3 , as a function of wave development. The absolute value of the weighting functions becomes smaller as the waves tend toward full development $(\Omega \rightarrow 0.84)$. When all these effects are combined, one should find that the low-order EM bias is always smaller (in absolute value) at the Ku band than at the $\mathrm{C}$ band. This agrees with measurements by Walsh et al. [1991] and Hevizi et al. [1.993].

\subsection{Sea State Bias}

In order to compare the first-order EM bias with empirical models derived from satellite data we need to compute the sea state bias. The sea state bias $\beta_{S S}$ is usually the measurable parameter, and it encompasses several biases. Among them we cite the EM bias, the elevation-skewness bias, and the tracker bias. The latter is usually neglected as present trackers are very stable. The second important bias after the electromagnetic bias is thus the elevation-skewness bias. This bias is introduced into the measurements by the onboard operational algorithms that use median estimators to estimate sea level from backscattered signals. When this statistical process is non-Gaussian, an additional bias is then introduced that consists of the difference between the mean and the median. In the present context the surface elevation is a perturbed Gaussian derived as a marginal pdl of (6) when the slope components are integrated out:

$$
P(\zeta)=\frac{G_{1-D}(\eta)}{\sqrt{\kappa_{2001}}}\left[1+\frac{\lambda_{3010}}{6} H_{3000}(\eta)\right] .
$$

The normalized median $M$ of a process with a predetermined pdf as in (42) is by definition

$$
\int_{-\infty}^{M} P(\zeta) d \zeta=\frac{1}{2}
$$

If the median $M$ and the mean $m=0$ are very close, (43) can be Taylor expanded about $m$ to give up to third order in $M$

$$
\begin{aligned}
& \int_{-\infty}^{m} P(\zeta) d \zeta+P(m) M+\frac{P^{\prime}(m)}{2} M^{2}+\frac{P^{\prime \prime}(m)}{6} M^{3}+O\left(M^{4}\right) \\
& \quad=\frac{1}{2}
\end{aligned}
$$

After a quick evaluation of the integral and derivatives of $P(\zeta)$ one finds a polynomial equation in $M$ through the third power:

$$
-\frac{\lambda_{300}}{6}+M+\frac{\lambda_{300}}{4} M^{2}-\frac{1}{6} M^{3}+O\left(M^{4}\right)=0 .
$$

The solution of this polynomial equation can be sorted in powers of $\lambda_{300}$ as it is considered the smallness parameter of the expansion:

$$
M={ }_{1} M+{ }_{2} M+{ }_{3} M+\ldots
$$

Replacing $M$ in (45) by (46) and sorting the orders of $M$ as powers of $\lambda_{300}$ one obtains,

$$
\begin{aligned}
{ }_{1} M & =\frac{\lambda_{300}}{6}, \\
{ }_{2} M & =0,
\end{aligned}
$$

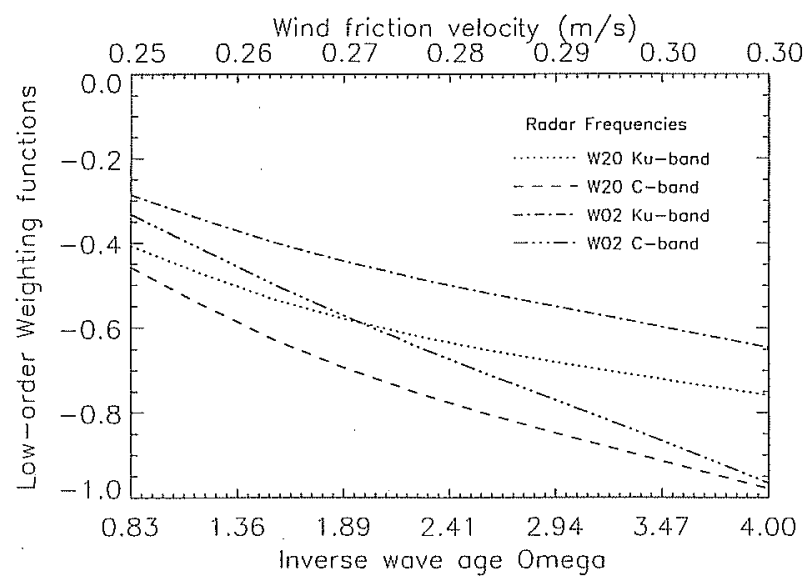

Figure 3. Same as Figure 2 but with a fixed separation taken to be 10 times the dominant peak frequency $\left(k_{s}=10 k_{p}\right)$. The inverse wave age is varied from 0.84 (fully developed seas) up to 4 (young seas) at a fixed wind speed of $U_{10}=7 \mathrm{~m} \mathrm{~s}^{-1}$. Note again that the magnitudes of the $\mathrm{Ku}$ band weighting functions are always larger than those for the $\mathrm{C}$ band. The absolute value of the weighting functions becomes smaller as the waves become older. 


$$
{ }_{3} M=\frac{5}{3}\left(\frac{\lambda_{300}}{6}\right)^{3}
$$

The normalized elevation skewness bias is then

$$
\gamma_{E S}=-\frac{1}{4}\left[\frac{\lambda_{300}}{6}+\frac{5}{3}\left(\frac{\lambda_{300}}{6}\right)^{3}+O\left(\lambda_{300}^{5}\right)\right] .
$$

Usually, the cubic term in (48) is neglected as the elevation skewness $\lambda_{300}$ is generally smaller than 0.25 [see, e.g., LonguetHiggins, 1963].

Figure 4 shows the normalized sea state bias by combining (48) and (24),

$$
\begin{aligned}
\gamma_{S S} & =\gamma_{E S}+\gamma_{E M} \\
& =\frac{1}{8}\left(-\frac{\lambda_{300}}{3}+\lambda_{120} W_{20}+\lambda_{102} W_{02}+2 \lambda_{111} W_{11}\right),
\end{aligned}
$$

as function of wind speed at a height of $10 \mathrm{~m}$. On Figure 4 we also plotted a simulation of the previous theory by Srokosz [1986] as simulated by Glazman et al. [1996]). Our theory seems to give closer agreement with the sea state bias data as reported by Gaspar et al. [1994] and Chelton [1994]. The reader is invited to notice, in addition to the difference in amplitude between our simulation and that of Srokosz [1986], the frequency sensitivity when the frequency changes from the $\mathrm{Ku}$ band to the $\mathrm{C}$ band. (The model of Srokosz does not predict such frequency dependence, thus the single solid curve in Figure 4.) For sake of simplicity the direction of shortwaves was taken to be identical to that of the long waves. A more complete sensitivity study is in progress to assess fully the effect of wind-wave directions as well as radar frequencies. Furthermore, short waves are not only tilted by long waves but also hydrodynamically modulated. In this case, statistical moments of short waves change dynamically with changes in the underlying long wave field. We are presently investigating the effects of such hydrodynamic modulation on the electromagnetic bias, which will be added in the future to this newly proposed theory.

\section{Conclusion}

A new theory on the electromagnetic bias (EM bias) has been derived by considering the nonlinear statistics of both short and long waves. Two separate sources of EM bias are identified. The first is the low-order EM bias, which depends on the cross skewness between the elevation and slopes weighted by ratios of slope variances of both short and long waves. This low-order EM bias may be understood as a modification to the previous theory by Srokosz [1986]. Indeed, Srokosz predicted the normalized EM bias to be solely dependent on the cross skewness between elevation and slopes. Since the weakly nonlinear theory developed by Longuet-Higgins [1963] cannot handle short waves [see Elfouhaily et al., 1999], Srokosz's theory is only applicable in the longwave domain. Our proposed modification accounts for the expected shortwave effects in the EM bias through the weighting functions. Scaling by these weighting functions reduces the longwave EM bias by a nonnegligible amount of the order of $50 \%$.

The second potential source of EM bias is the high-order contribution that involves the slope skewness of short waves and the cross kurtosis together with weighting functions of the slope variances. We believe that this is the first time these high-order moments have been introduced into the EM bias

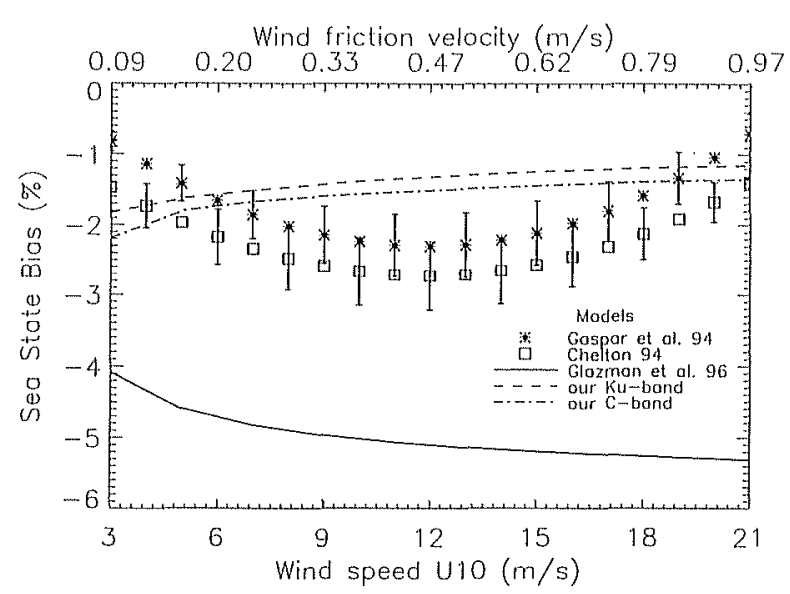

Figure 4. The sea state bias as obtained by combining our low-order EM bias and the elevation-skewness bias (dashed $\mathrm{Ku}$ band and dash-dotted $\mathrm{C}$ band). The previous formulation by Srokosz [1986] is plotted with the solid curve. Comparison with data is also provided through empirical models by Gaspar et al. [1994] (stars) and Chelton [1.994] (squares) derived from data taken by the TOPEX altimeter. Difference in the propagation direction between short and long waves is not considered. Swell is also not included in these simulations regardless of its influence on the electromagnetic bias. It must be noted that only tilt modulations are modeled, which means that for instance, hydrodynamic interactions between short and long waves are not yet included.

theory. The shortwave effects are no longer limited to the slope variances but also enter the EM bias through "nonlinear" moments: the slope skewness. Both low and high orders can be combined to form a novel theory for the EM bias.

In addition to the variances and the nonlinear moments, the cross-correlation moments are important in the general twodimensional problem. Indeed, our new formulation encompasses two separate cross correlations. Since the crosscorrelation moments are mainly generated by differences in the directions between the waves and the principal axis, it is clear that directions of both short and long waves must be treated separately. Only rarely is the propagation direction of the short waves aligned with that of long waves. This makes the presence of two different cross-correlation moments critically important. The inclusion of two separate cross-correlation moments is another key feature of our two-dimensional formulation.

Another powerful feature of our new formulation is the sensitivity to the radar frequency. Indeed, several investigators have suggested in the past that the geometric optics approximation leaves the EM bias theory insensitive to the EM frequency. Consequently, more complicated EM models, such as the physical optics approximation, were invoked in efforts to recover the frequency dependence. It is now clear from our formulation that the frequency dependence enters the EM bias through the presence of short wave slope variance in the model. A geometric optics approximation can thus provide frequency dependence under our assumption that short waves are modulated by long waves.

It is expected that the low-order correction presented in this paper is already of major importance since it introduces three significant contributions. When all of these effects are combined, we have shown that closer agreement with measured 
EM bias by the TOPEX altimeter can be obtained. In addition, one can take advantage of our formulation by simply incorporating measured surface moments to reproduce a statistical description of the EM bias. A sensitivity study of the EM bias on measured and simulated surface moments is also planned for future work.

\section{Appendix A: Generalized Two-Dimensional Hermite Polynomials}

The one- and two-dimensional Gaussian distributions are abbreviated as

$$
\begin{gathered}
G_{1-\mathrm{D}}(\eta)=\frac{1}{\sqrt{2 \pi}} e^{-\eta^{2} / 2} \\
G_{2-\mathrm{D}}(x, y ; \rho)=\frac{1}{2 \pi \sqrt{1-\rho^{2}}} e^{-x^{2}-2 \mu y+y^{2} / 2\left(1-\rho^{2}\right)}
\end{gathered}
$$

where the mean values are zero and the variances are normalized to unity. The generalized Hermite polynomials are defined as normalized derivatives of the two-dimensional Gaussian distributions [e.g., Longuet-Higgins, 1963, p. 474]:

$$
\begin{aligned}
& H_{i j}(x, y ; \rho) \\
& \quad=(-1)^{i+j}\left[G_{2-\mathrm{D}}(x, y ; \rho)\right]^{-1} \frac{\partial^{i+j}}{\partial x^{i} \partial y^{j}}\left[G_{2-\mathrm{D}}(x, y ; \rho)\right] .
\end{aligned}
$$

The degree of a generalized Hermite polynomial is defined as the sum of its two indices. Therefore generalized Hermite polynomials of degree $m$ include all the polynomials $H_{i j}$ with $i+j=m$. We will only give the explicit form of the generalized Hermite polynomials required for the evaluation of both the low- and high-order electromagnetic bias.

The first degree polynomials are

$$
\begin{gathered}
H_{10}(x, y ; \rho)=\frac{x-\rho y}{1-\rho^{2}} \\
H_{01}(x, y ; \rho)=\frac{y-\rho x}{1-\rho^{2}} .
\end{gathered}
$$

Since a permutation in the indices always means a simple interchange of the $x$ and $y$ components, we need to discuss only one asymmetric polynomial.

The low-order electromagnetic bias involves second-degree polynomials,

$$
\begin{aligned}
& H_{20}(x, y ; \rho)=H_{10} H_{10}-\frac{1}{1-\rho^{2}} \\
& H_{11}(x, y ; \rho)=H_{10} H_{01}+\frac{\rho}{1-\rho^{2}} .
\end{aligned}
$$

Third-degree polynomials are required for the computation of the higher-order electromagnetic bias:

$$
\begin{array}{r}
H_{30}(x, y ; \rho)=H_{10}^{2} H_{10}-\frac{3}{1-\rho^{2}} H_{10} \\
H_{21}(x, y ; \rho)=H_{10}^{2} H_{01}+\frac{2 \rho}{1-\rho^{2}} H_{10}-\frac{1}{1-\rho^{2}} H_{01} .
\end{array}
$$

This is the first time that these third-degree polynomials are integrated into the theory of the EM bias.

\section{Appendix B: Some Required Identities}

Let us define the following double integral that involves two generalized Hermite polynomials of arbitrary degrees. Further, let $l_{i j}^{k l}(\rho)$ be that quantity that is a function of the crosscorrelation or coupling parameter $\rho$ :

$$
I_{i j}^{k l}(\rho)=\iint G_{2-13}(x, y ; \rho) H_{i j}(x, y ; \rho) H_{k l}(x, y ; \rho) d x d y .
$$

After a trivial change of variable in order to decouple the generalized two-dimensional Gaussian distribution we obtain

$$
\begin{aligned}
I_{i j}^{k l}(\rho)= & \iint G_{1-\mathrm{D}}(X) G_{1-\mathrm{D}}(Y) H_{i j}\left(X \sqrt{1-\rho^{2}}+\rho Y, Y ; \rho\right) \\
& \cdot H_{k l}\left(X \sqrt{1-\rho^{2}}+\rho Y, Y ; \rho\right) d X d Y .
\end{aligned}
$$

One can easily verify the following identities:

$$
\begin{gathered}
I_{10}^{10}(\rho)=\frac{1}{1-\rho^{2}}, \\
I_{20}^{20}(\rho)=\frac{2}{\left(1-\rho^{2}\right)^{2}}, \\
I_{30}^{30}(\rho)=\frac{6}{\left(1-\rho^{2}\right)^{3}}, \\
I_{11}^{11}(\rho)=\frac{1+\rho^{2}}{\left(1-\rho^{2}\right)^{2}}, \\
I_{21}^{21}(\rho)=\frac{2\left(1+2 \rho^{2}\right)}{\left(1-\rho^{2}\right)^{3}} .
\end{gathered}
$$

It should be emphasized that the generalized Hermite polynomials are not orthogonal if the coupling parameter is not zero. This means that some cross products of the Hermite polynomials with nonmatching indices may survive the double integration in (B2). We identified only two nonzero cross products. They are

$$
\begin{aligned}
& I_{20}^{11}(\rho)=-\frac{2 \rho}{\left(1-\rho^{2}\right)^{2}} \\
& I_{30}^{21}(\rho)=-\frac{6 \rho}{\left(1-\rho^{2}\right)^{3}} .
\end{aligned}
$$

The unlisted cross products are zero even if the coupling parameter is not.

\section{Appendix C: Derivation of the Higher-Order EM Bias}

The low-order EM bias is already given in its general form in (24) and (25). This general form is shown to be understood as a modification of Srokosz's theory. We have even demonstrated that Srokosz's theory is actually a special case of the current low-order theory when shortwave effects are neglected. Now we will show the derivation of the higher-order EM bias, which in turn can be understood as an extension of Srokosz's theory. The higher-order EM bias can be isolated and written as

$$
\begin{aligned}
\gamma_{H}= & \frac{1}{8}\left(\lambda_{30} \lambda_{130} W_{30}^{30}+\lambda_{03} \lambda_{103} W_{03}^{03}+\lambda_{21} \lambda_{121} W_{21}^{21}+\lambda_{12} \lambda_{112} W_{12}^{12}\right. \\
& \left.+\lambda_{30} \lambda_{121} W_{30}^{21}+\lambda_{03} \lambda_{112} W_{03}^{12}+\lambda_{21} \lambda_{130} W_{21}^{30}+\lambda_{12} \lambda_{103} W_{12}^{03}\right)
\end{aligned}
$$


where all the nonzero weighting functions are retained. Some of the cross terms exist because of the fact that the generalized Hermite polynomials are not fully orthogonal when the coupling coefficient is different from zero (see Appendix B). The weighting functions at this high order are given in terms of a double integral of the product of two generalized Hermite polynomials. We give hereafter the direct expression of the double integral subsequent to a change of variable in order to decouple the two-dimensional Gaussian distribution:

$$
\begin{aligned}
W_{i j}^{k l}= & \frac{1}{i ! j !} \iint G_{1-\mathrm{D}}(X) G_{1-\mathrm{D}}(Y) \\
& \cdot H_{i j}\left[\Delta_{x}^{L}\left(X \sqrt{1-R^{2}}+R Y\right), \Delta_{y}^{L} Y ; \lambda_{011}\right] \\
& \cdot H_{k l}\left[\Delta_{x}^{S}\left(X \sqrt{1-R^{2}}+R Y\right), \Delta_{y}^{S} Y ; \lambda_{11}\right] d X d Y .
\end{aligned}
$$

The superscripts $L$ and $S$ over the deltas refer to the normalization by the slope rms of long and short waves, respectively. We should stress that these superscripts do not mean that the respective deltas depend only on the long or short waves. In fact, each delta is a function of both longwave and shortwave parameters. Actually, they define a measure of the coupling between long and short waves [see Elfouhaily, 1996, paragraph III.6].

Analytical evaluation of (C2) is not an easy task, although the procedure is similar to the one already explained for the lower-order weighting functions. We propose to write the final expression of $W_{i j}^{k l}$ as a quadruple sum of the product of two generalized Hermite polynomials. The compact form of these weighting functions would look like

$$
\begin{aligned}
W_{i j}^{k l}= & \frac{1}{i ! j !} \\
& \cdot \sum a_{m n}^{u v}\left(\lambda_{011}, \lambda_{11}, R\right) H_{m n}\left(\Delta_{x}^{L}, \Delta_{y}^{L} ; \lambda_{011}\right) H_{u v}\left(\Delta_{x}^{S}, \Delta_{y}^{S} ; \lambda_{11}\right),
\end{aligned}
$$

where the sum is performed over all the combination of indices that satisfy the inequality $(m+n+u+v) \leq(i+j+k+$ l).

For our development of the higher-order electromagnetic bias we need only two asymmetric weighting functions that do not vanish when the cross correlations $\lambda_{011}$ and $\lambda_{11}$ are zero. The required weighting functions are

$$
\begin{aligned}
W_{30}^{30}= & \frac{1}{6}\left(a_{30}^{30} H_{30}^{L} H_{30}^{S}+a_{30}^{10} H_{30}^{L} H_{10}^{S}+a_{30}^{21} H_{30}^{L} H_{21}^{S}+a_{10}^{10} H_{10}^{L} H_{10}^{S}\right. \\
& \left.+a_{10}^{21} H_{10}^{L} H_{21}^{S}\right)+(S \stackrel{A}{\rightleftharpoons} L) \\
W_{21}^{21}= & \frac{1}{2}\left(b_{21}^{21} H_{21}^{L} H_{21}^{S}+b_{21}^{01} H_{21}^{L} H_{01}^{S}+b_{21}^{30} H_{21}^{L} H_{30}^{S}+b_{01}^{01} H_{01}^{L} H_{01}^{S}\right) \\
& +(S \stackrel{A}{\rightleftharpoons} L),
\end{aligned}
$$

where $A$ over the double arrows means that the interchange of $L$ and $S$ in is executed over asymmetric polynomials only. The as of (C4a) and (C4b) are in their general form given as follows:

$$
\begin{gathered}
a_{30}^{30}=5 \\
a_{30}^{10}=\frac{12+6 \lambda_{11}^{2}\left(1-5 R+2 R^{2}\right)}{\left(1-\lambda_{11}^{2}\right)^{2}}
\end{gathered}
$$

$$
\begin{gathered}
a_{30}^{21}=-15 R \frac{\lambda_{11}}{1+2 \lambda_{11}^{2}}, \\
a_{111}^{111}=\frac{30+6 P_{2}(R)\left(\lambda_{11}^{2}+\lambda_{011}^{2}\right)+6 P_{3}(R) \lambda_{011}^{2} \lambda_{11}^{2}}{\left(1-\lambda_{011}^{2}\right)^{2}\left(1-\lambda_{11}^{2}\right)^{2}}, \\
a_{111}^{21}=-18 \lambda_{11} \frac{2 R+\left(-1+2 R-4 R^{2}+R^{3}\right) \lambda_{011}^{2}}{\left(1-\lambda_{011}^{2}\right)^{2}\left(1+2 \lambda_{11}^{2}\right)} .
\end{gathered}
$$

The $b$ s are similarly given as

$$
\begin{gathered}
b_{21}^{21}=3\left(1+4 R^{2}\right), \\
b_{21}^{01}=2 \frac{1+5 R^{2}+Q_{3}(R) \lambda_{S}^{2}+T_{3}(R) \lambda_{S}^{4}}{\left(1-\lambda_{11}^{2}\right)^{2}}, \\
b_{21}^{30}=-15 R \lambda_{11}, \\
b_{01}^{01}=2 \frac{1+R P_{4}\left(\lambda_{110} ; R\right) P_{4}\left(\lambda_{11} ; R\right)}{\left(1-\lambda_{011}^{2}\right)^{2}\left(1-\lambda_{11}^{2}\right)} .
\end{gathered}
$$

The parameters $P_{2}, P_{3}, Q_{3}$, and $T_{3}$ are third-order polynomials of the generalized coupling parameter $R$. The explicit expression of these third-order polynomials as well as the fourth-order one $\left(P_{4}\right)$ will not be given in this paper for sake of simplicity. It can readily be seen that these polynomials are not needed if both cross correlations $\lambda_{011}$ and $\lambda_{11}$ are set to zero. The $a \mathrm{~s}$ and $b \mathrm{~s}$ from these equations were used to generate the applied constants in the expression of the EM bias (equation (39)).

Since the analytical evaluation of these generalized weighting functions is a complicated task, we have decided to give the analytical expressions of the weighting functions that are nonzero when both $\lambda_{011}$ and $\lambda_{11}$ go to zero. For the more general case the expressions are much more difficult to derive. In the future we plan to use an algebraic software package such as Mathematica or Maple to find error-free analytical solutions of the high-order EM bias.

Acknowledgments. We would like to thank our colleagues in the Ocean Remote Sensing Group of the Applied Physics Laboratory at Johns Hopkins University for the helpful comments. Among them, special thanks go to Keith Raney for contributing significantly to the clarity of this paper.

\section{References}

Apel, J., An improved model of the ocean surface wave vector spectrum and its effect on radar backscatter, J. Geophys. Res., 99, 16,269$16,291,1994$

Arnold, D., W. Melville, and J. Kong, Theoretical prediction of em bias, in $O C E A N S^{\prime} 90$, Engineering in the Ocean Environment, Oceanic Eng. Soc. Inst., Washington, D. C., 1990.

Arnold, D., J. Kong, and W. Melville, Physical optics prediction of EM bias, in Progress in Electrontagnetic Research, IEEE Press, Piscataway, N. J., 1991.

Barrick, D., and B. Lipa, Analysis and interpretation of altimeter sea echo, Adv. Geophys., 27, 60-99, 1985.

Barrick, D., and B. Weber, On the nonlinear theory for gravity waves on the ocean's surface, II, Interpretation and applications, $J$. Phys. Oceanogr., 7, 11-21, 1977.

Brown, G., Backscattering from a Gaussian distributed perfectly conducting rough surface, IEEE Trans. Antennas Propag., 26, 472-482, 1978.

Chapron, B., K. Katsaros, T. Elfouhaily, and D. Vandemark, A note on relationships between sea surface roughness and altimeter backscatter, in Air-Water Gas Transfer, edited by B. Jähne and E. C. Monahan, pp. 869-878, AEON Verlag, Heidelberg, Germany, 1995. 
Chelton, D., The sea state bias in altimeter estimates of sea level from collinear analysis of TOPEX data, J. Geophys. Res., 99, 24,995$25,008,1994$

Cox, C., and W. Munk, Statisties of the sea surlace derived from Sun glitter, J. Mar: Res., 13, 198-227, 1954.

Donelan, M., and W. Pierson, Radar scattering and equilibrium ranges in wind-generated waves with application to scatterometry, J. Geophys. Res., 92, 4971-5029, 1987.

Elfouhaily, T., A consistent wind and wave model and its application to microwave remote sensing of the ocean surface, Ph.D. thesis, Univ. de Denis Diderot, Paris, 1996.

Elfouhaily, T., B. Chapron, K. Katsaros, and D. Vandemark, A unified directional spectrum for long and short wind-driven waves, J. Geoplys. Res., 102, 15,781-15,796, 1997.

Elfouhaily, 'T., D. Thompson, B. Chapron, and D. Vandemark, Weakly' nonlinear theory and sea state bias estimations, J. Geophys. Res., 104, $7641-7647,1999$

Elfouhaily, T., D. Thompson, B. Chapron, and D. Vandemark, Truncated Hamiltonian versus surlace perturbation in nonlinear wave theories, J. Fluid Mech., in press, 2000.

Gaspar, P., F. Ogor, P.-Y. L. Traon, and O.-Z. Zanifé, Estimating the sea state bias of the TOPEX and Poseidon altimeters from crossover differences, J. Geophys. Res., 99, 24,981-24,994, 1.994.

Glazman, R., and M. Srokosz, Equilibrium wave spectrum and sea state bias in altimetry, J. Plys. Oceanogr., 21, 1609-1621, 1991.

Glazman, R., A. Fabrikant, and M. Srokosz, Numerical analysis of the sea state bias for satellite altimetry, J. Geophys. Res., 101, 3789-3799, 1996.

Hasselmann, K., On the non-linear energy transfer in gravity-wave spectrum, 1, General theory, J. Fluid Mech., 12, 481-500, 1961.

Hevizi, L., E. Walsh, R. Maclntosh, D. Vandemark, D. Hines, R. Swift, and J. F. Scott, Electromagnetic bias in sea surlace range measurements at frequencies of the TOPEX/Poseidon satellite, IEEE Trans. Geosci. Remote Sens., 31, 367-388, 1993.

Jackson, F., The reflection of impulses from a non-linear random sea, J. Geophys. Res., 84, 4939-4943, 1979.

Keller, W., and J. Wright, Microwave scattering and the straining of wind generated waves, Radio Sci., 10, 139-147, 1975.

Kinsman, B., Surface wave at short fetches and low wind speed: A field study, Tech. Rep. 19, Chesapeake Bay Inst., Annapolis, Md., 1960.

Lagerloef, G., Comment on "On the joint clistribution of surface elevation and slopes for a nonlinear random sea, with an application to radar altimetry" by M. A. Srokosz, J. Geophys. Res., 92, 2985-2987, 1987.
Longuet-1-1iggins, $M$., The effects of non-linearities on statistical distribution in the theory of sea watves, J. Fluid Mech., 17, 459-480, 1963.

Nouel, F., el al., Precise Centre National d'Eucles Spatiales orbits for TOPEX/Poseidon: Is reaching $2 \mathrm{~cm}$ still a challenge?, $/$. Geophys. Res., 99, 24,405-24,419, 1994.

Rodriguez, E., Y. Kim, and J. M. Martin, The effect of small-wave modulation on the electromagnetic bias, J. Geophys, Res., 97, 2379$2389,1992$.

Srokosz, M., On the joint distribution of surface elevation and slopes for a non-linear random sea, with an application to radar altimetry, J. Geophys. Res., 91, 995-1006, 1986.

Srokosz, M., Reply to comment by C.S.E. Lagerloef on "on the joint distribution of surface elevation and slopes for a nonlinear random sea, with an application to radar altimetry," I. Geophys. Res., 92, 2989-2990, 1987

Thompson, D., Calculation of radar backscatter modulations from internal waves, J. Geophys. Res., 93, 12,371-12,380, 1988.

Valenzuela, G., Theories for the interaction of electromagnetic and oceanic waves: A review, Boundayy Layer Meteorol., 13, 61-85, 1978.

Walsh, E., F. C. Jackson, D. Hines, C. Piazza, and L. Hevizi, Frequency dependence of electromagnetic bias in radar altimeter sea surface, $J$ Geophys. Res., 96, 571-583, 1991.

Yaplee, B., A. Shapiro, D. Hammond, B. Au, and E. Uliana, Nanosecond radar observations of the ocean surface from a stable platform, IEEE Trans. Geosci. Electron., 9, 170-174, 1971.

Zakharov, V. E., Stability of periodic waves of finite amplitude on the surlace of a deep fluid, J. Appl. Mech. Tech. Phys. Engl. Thansl., 2, $190-194,1968$.

B. Chapron, Départment d'Océanographie Spatiale, IFREMER Centre de Brest, BP 70, 29280 Plotzané, France. (Bertrand.Chapron@ ifremer.fr)

T. Elfouhaily and D. R. Thompson, Applied Physics Laboratory, Johns Hopkins University, 11100 Johns Hopkins Road, Laurel, MD 20723-6099. (Elfouhaily@jhuapl.edu; Donald.Thompson@jhuapl.edu)

D. Vandemark, Laboratory for Hydrospheric Processes, NASA Goddard Space Flight Center, Wallops Island, VA 23337. (vandemark@ gsfc.nasa.gov)

(Received March 9, 1999; revised September 21, 1999; accepted September 28, 1999.) 